\title{
La métallurgie du fer dans les Deux-Sèvres et la Vienne
}

\section{Guillaume Saint-Didier}

\section{OpenEdition Journals}

Édition électronique

URL : http://journals.openedition.org/adlfi/1237

ISSN : 2114-0502

Éditeur

Ministère de la culture

Référence électronique

Guillaume Saint-Didier, "La métallurgie du fer dans les Deux-Sèvres et la Vienne », ADLFI. Archéologie de la France - Informations [En ligne], Poitou-Charentes, mis en ligne le 01 mars 2008, consulté le 03 mai 2019. URL : http://journals.openedition.org/adlfi/1237

Ce document a été généré automatiquement le 3 mai 2019.

(c) Ministère de la Culture et de la Communication, CNRS 


\title{
La métallurgie du fer dans les Deux- Sèvres et la Vienne
}

\author{
Guillaume Saint-Didier
}

Identifiant de l'opération archéologique : 204479

Date de l'opération : 2008 (PT)

1 Les prospections archéologiques pédestres menées au cours de l'année 2008 dans les départements des Deux-Sèvres et de la Vienne s'inscrivent dans le cadre de ma thèse préparée à l'université de Poitiers sous la direction de Nadine Dieudonné-Glad, dont le titre est «La métallurgie du fer à l'époque romaine chez les Pictons ». Cette thèse a été débutée à la fin de l'année 2007 et cette campagne de prospections qui vient de s'achever est donc la première de cette thèse.

2 Le but de ces prospections était de repérer un maximum de sites archéologiques liés au travail du fer. On connaissait auparavant près de six cents ferriers dans la moitié sud de la Vienne (la quasi totalité repérés par Christian Richard lors de ses prospections aériennes dans les années 1990), mais on ne connaissait qu'un total de 23 sites liés à cette activité dans les Deux-Sèvres ( 1 site d'extraction du minerai de fer, 14 ferriers (dont 4 ne sont pas localisés précisément), 3 forges fouillées dont 2 sont gallo-romaines, 2 probables forges domestiques, 1 remploi de scories issues d'un atelier de travail du fer et 2 sites où des scories ont été trouvées sans pour autant qu'il s'agisse de ferriers).

Environ la moitié de ceux-ci étaient situés dans les vallées du Thouet et du Thouaret ou en bordure immédiate. Pour la partie deux-sévrienne, ces prospections avaient donc comme principal objectif d'évaluer le potentiel archéologique par rapport à la métallurgie $\mathrm{du}$ fer de la zone comprise entre Thouars, Parthenay et en moindre mesure Bressuire. Un total de 40 sites archéologiques y ont été découverts, dont 36 sites sont effectivement liés à la métallurgie du fer. Parmi ces 36 sites, 19 sont des sites sûrs et certains, 4 sont possibles et les 12 autres sont plus problématiques, compte tenu qu'il s'agit de creusements situés dans la forêt d'Autun. En effet, cette forêt est mentionnée en 1921 et 
en 1928 par l'abbé Vigué comme ayant de nombreux creusements dus à l'extraction du minerai de fer par le passé, mais malheureusement, elle a été bombardée durant la Seconde Guerre Mondiale. Or, il est très difficile de différencier un trou d'extraction du minerai d'un trou d'obus, sauf si l'on trouve en bordure un fragment de minerai (la végétation était trop importante pour pouvoir faire cette observation cette année).

Les résultats sont très encourageants, puisqu'il semble que cette zone puisse être ce qu'on appelle, à la suite de Michel Mangin, "un district sidérurgique ». C'est donc une découverte très importante au sujet de l'étude de la métallurgie du fer sur le territoire picton. Il semblerait que cette zone compte davantage de sites dans la moitié orientale, avec notamment de fortes concentrations de sites autour de Thouars - Saint-Léger-deMontbrun d'un côté et de Thénezay et la forêt d'Autun de l'autre. Néanmoins, il y a là peut-être un effet de source puisqu'il est beaucoup plus facile de prospecter dans les plaines céréalières du Thouarsais que dans les prairies du Bocage Bressuirais. Les sites de cette zone ayant pu être datés remontent à la période gallo-romaine. Aucun site n'est protohistorique et aucun n'est médiéval. Beaucoup restent sans datation.

De plus, le secteur allant de Saint-Maixent-l'École à la forêt de Saint-Sauvant (86), en passant par le village de Rom (antique agglomération secondaire de Rauranum), semble être également très riche en ferriers. Peu ont été prospectés (trois) (on peut ajouter le site publié de Vautebis mais non prospecté cette année), mais d'après le témoignage d'agriculteurs de ce secteur, il y aurait des scories partout et en grande quantité. Il conviendra donc de poursuivre l'année prochaine à la fois dans ce secteur et dans celui des vallées du Thouet et du Thouaret, et d'élargir ces deux zones afin de voir jusqu'où elles vont et si elles restent distinctes ou s'il ne s'agit que d'une seule zone pouvant être reliée à celle du sud de la Vienne.

6 Pour la Vienne, la campagne a consisté à prospecter des sites déjà connus afin de pouvoir les dater et les caractériser. Beaucoup sont gallo-romains ; les autres étant médiévaux ou non datés, faute de découverte de matériel archéologique adéquat. vingt-huit sites ont été prospectés dans la Vienne, dont 20 repérés par Christian Richard. Parmi les 8 sites restants, 1 n'est pas situé dans la zone sidérurgique

du sud de la Vienne (Villiers), 1 est situé en limite de cette zone (Saint-Sauvant), 1 est le prolongement d'un site découvert par C. Richard (Usson-du-Poitou, site 011), et les 5 autres sont situés dans le district sidérurgique du sud de la Vienne.

Le but de ces prospections, à terme, est de pouvoir retracer toute l'économie du fer à l'époque romaine sur le territoire des Pictons. Pour atteindre cet objectif, de nombreuses scories et de nombreux fragments de minerai ainsi que de parois de fours ont été prélevés sur les sites prospectés afin de procéder à des analyses chimiques ayant pour but de caractériser la composition chimique de ces éléments afin de pouvoir relier les sites d'extraction de minerai aux ateliers de travail du fer. 
INDEX

operation Prospection thématique (PRT)

Thèmes : acquisition de ressource naturelle, analyse des matériaux, atelier métallurgique, fer, ferrier, forge, four, métallurgie, minerai, scories, site d'extraction

Index géographique : Poitou-Charentes

Index chronologique : Empire romain, Moyen Âge*

\section{AUTEURS}

GUILLAUME SAINT-DIDIER

DOC 